\title{
A DISCRETE STOCHASTIC KOROVKIN THEOREM
}

\author{
GEORGE A. ANASTASSIOU \\ Department of Mathematical Sciences \\ Memphis State University \\ Memphis, Tennessee 38152 U.S.A. \\ (Received June 2, 1989 and in revised form October 16, 1989)
}

ABSTRACT. In this article we give a sufficient condition for the pointwise -- in the first mean Korovkin property on $B_{0}(P)$, the space of stochastic processes with real state space and countable index $\overline{s e t} \Gamma$ and bounded first moments.

KEY WORDS AND PURASES. Positive linear operator, stochastic processes, pointwise - in the first mean convergence.

1980 AMS SUBJECT CLASSIFICATION CODE. (1985 Revision): Primary 41^36, 60F 25;

Secondary 60C99.

1. INTRODUCTION.

Let $(\underline{0}, A, \tau)$ be a probability space and let $P$ denote a fixed countable set. Consider stochastic processes $X$ with real state snace and the expectation operator $E(X)(t)=\int_{\underline{0}} X(t, \omega) \tau(d \omega), t \in P$. Define $B_{\underline{0}}(P)=\left\{X: \sup _{t \in P} E|X|(t)<\infty\right\}$. Let $T_{n}$ : $\mathrm{B}_{0}(P) \rightarrow \mathrm{B}_{0}(P)$ be any sequence of positive linear operators such that $\mathrm{ET}_{n}=T_{n} E$, all $n=1,2, \ldots$. In Theorem 1 , under Korovkin type assumptions, we give a sufficient condition such that for each $X \in B_{\underline{0}}(P)$,

$$
\lim _{n \rightarrow \infty} E\left[\left(T_{n} X\right)(t, \omega)-X(t, \omega)\right]=0 \text {, for each } t \in P .
$$

In [3], see Theorem 3.2, was treated the continuous case, that is, when $P$ is an uncountable compact space. There the sufficient condition is similar to ours, however, it is produced under the additional assumption that $T_{n}$ is a stochastically simple operator.

Our result has as follows:

THEOREM 1. Let $(\underline{0}, A, \tau)$ be a probability space and $\Gamma=\left\{t_{1}, \ldots, t_{j}, \ldots\right\}$ be a countable set of cardinality $\geq 2$. Consider the space of stochastic processes with real state space

$$
B_{\underline{0}}(P)=\left\{X: \sup _{t \varepsilon P} \int_{0}|X(t, \omega)| \tau(d \omega)<\infty\right\}
$$

and the space

$$
B(P)=\left\{f:\left.P \rightarrow \mathbb{R}|\| f|\right|_{\infty}<\infty\right\},
$$

where

$$
|| f||_{\infty}=\sup _{t \in P}|f(t)| ; B(P) \subset B_{0}(P) .
$$

Let $T_{n}: B_{0}(P) \rightarrow B_{0}(P)$ be a sequence of positive linear operators that are E-commutative, i.e.

$$
\left(E\left(T_{n} X\right)\right)(t, \omega)=\left(T_{n}(E X)\right)(t, \omega), \text { for all }(t, \omega) \varepsilon P \times \underline{0}
$$


where

is the expectation.

$$
(E X)(t):=E(X(t, \omega)):=\int_{\underline{0}} X(t, \omega) \tau(d \omega)
$$

Also assume that $\left(T_{n} 1\right)(t, \omega)=1$, for all $(t, \omega) \varepsilon \Gamma \times \underline{0}$. For

$$
\left\{x_{1}(t, \omega), \ldots, x_{k}(t, \omega)\right\} \subset B_{0}(P)
$$

assume that

$$
\lim _{n \rightarrow \infty} E\left[\left(T_{n} x_{i}\right)\left(t_{j}, w\right)-x_{i}\left(t_{j}, w\right)\right]=0 \text {, }
$$

for all $t_{j} \in P$ and all $i=1, \ldots, k$. (I.e.

$$
\lim _{n \rightarrow \infty}\left[\left(T_{n}\left(E X_{i}\right)\right)\left(t_{j}\right)-\left(E X_{i}\right)\left(t_{j}\right)\right]=0 \text {, }
$$

for all $t_{j} \in P$ and $i=1, \ldots, k$. )

In order that

$$
\lim _{n \rightarrow \infty} E\left[\left(T_{n} X\right)\left(t_{j}, \omega\right)-X\left(t_{j}, \omega\right)\right]=0,
$$

for all $t_{j} \in P$ and all $X \in B_{0}(P)$, it is enough to assume that each $t_{j} \varepsilon P$ there are real constants $\beta_{1}, \ldots, \beta_{k}$ such that

$$
\sum_{i=1}^{k} \beta_{i} E\left[x_{i}(t, \omega)-x_{i}\left(t_{j}, \omega\right)\right] \geq 1 \text {, for all } t \varepsilon P-\left\{t_{j}\right\} \text {. }
$$

PROOF. If there exists $X \in B_{0_{0}}(P)$ and $t_{j_{0}} \varepsilon P$ such that

$$
E\left[\left(T_{n} x\right)\left(t_{j_{0}}, \omega\right)-x\left(t_{j_{0}}, \omega\right)\right]+0,
$$

then there exist a subsequence $T_{\lambda_{n}}$ and an $\varepsilon>0$ such that

$$
\left|\left(E\left(T_{\lambda_{n}} X\right)\right)\left(t_{j_{0}}\right)-(E X)\left(t_{j_{0}}\right)\right|>\varepsilon \text {, for a11 } n \geq 1 \text {. }
$$

By E-commutativity of $T_{\lambda_{n}}$ we get

$$
\left|\left(T_{\lambda_{n}}(E X)\right)\left(t_{j_{0}}\right)-(E X)\left(t_{j_{0}}\right)\right|>\varepsilon \text {, for all } n \geq 1 \text {. }
$$

Let $\mu$ be a positive finite measure on $P$ with $\mu(\{t\})>0$, for all $t \varepsilon P$. Here $B(P) \subset L_{p}(P, \mu), 1 \leq p<\infty$.

Let $f \in B(P)$, then $E(f)=f$. Hence $T_{n}(f)=T_{n}(E f)=E T_{n}(f)$ and $T_{n}(f) \varepsilon B(P)$, i.e. $T_{n}$ maps $B(P)$ into itself. Because each positive linear functional $T_{n}\left(\cdot, t_{j}\right)$ on $B(P)$ is bounded, by Riesz representation theorem, for the specific $j=j_{0}$, there exists $g_{t_{j} \text {, }} \varepsilon L_{q}(P, \mu)$ where $\frac{1}{p}+\frac{1}{q}=1$ such that

$$
\left(T_{n}(f)\right)\left(t_{j_{0}}\right)=\int_{P} f(t) g_{t_{j} \delta^{n}}(t) \mu(d t) \text {, for all } f \in B(P) \text {. }
$$

By $T_{n}(1)=1$ and the positivity of $T_{n}\left(\cdot, t_{j_{0}}\right)$ one obtains

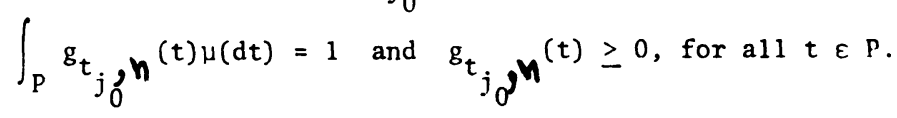

Since $\operatorname{EX} \varepsilon B(P)$, we have

$$
\left(T_{\lambda_{n}}(E X)\right)\left(t_{j_{0}}\right)=\int_{P}(E X)(t) \cdot g_{t_{j_{0}}, \lambda_{n}}(t) \cdot \mu(d t) .
$$


Thus

so that

$$
\begin{aligned}
& \varepsilon<\left|\left(T_{\lambda_{n}}(E X)\right)\left(t_{j_{0}}\right)-(E X)\left(t_{j_{0}}\right)\right|=\mid \int_{p}(E X)(t) \cdot g_{t_{j_{0}}, \lambda_{n}}(t) \cdot \mu(d t) \\
& -\int_{P}(E X)\left(t_{j_{0}}\right) \cdot g_{t_{j_{0}}, \lambda_{n}}(t) \cdot \mu(d t) \mid \\
& =\left|\int_{P-\left\{t_{j_{0}}\right\}}\left[(E X)(t)-(E X)\left(t_{j_{0}}\right)\right] \cdot g_{t_{j_{0}, \lambda_{n}}}(t) \cdot \mu(d t)\right| \\
& \leq\left\|E X-(E X)\left(t_{j_{0}}\right)\right\|_{\infty} \cdot\left(\int_{\Gamma-\left\{t_{j_{0}}\right\}} g_{t_{j_{0}, \lambda_{n}}}(t) \mu(d t)\right) \text {, }
\end{aligned}
$$

$$
\int_{\mathrm{P}-\left\{\mathrm{t}_{j_{0}}\right\}} \mathrm{g}_{\mathrm{t}_{0}, \lambda_{\mathrm{n}}}(\mathrm{t}) \mu(\mathrm{d} t)>\frac{\varepsilon}{\prod \mathrm{EX}-(\mathrm{EX})\left(\mathrm{t}_{\mathrm{j}_{0}}\right) \prod_{\infty}}=: \delta>0 \text {, for all } \mathrm{n} \geq 1 \text {. }
$$

There cannot be real constants $\beta_{1}, \ldots, \beta_{k}$ with

$$
\sum_{i=1}^{k} \beta_{i} E\left[x_{i}(t, \omega)-x_{i}\left(t_{j_{0}}, \omega\right)\right] \geq 1 \text {, for all } t \varepsilon p-\left\{t_{j_{0}}\right\} \text {. }
$$

Since, otherwise, we would have

$$
\sum_{i=1}^{k} \beta_{i} E\left[X_{i}(t, \omega)-x_{i}\left(t_{j_{0}}, \omega\right)\right] \cdot g_{t_{j_{0}}, \lambda_{n}}(t) \geq g_{t_{j_{0}}, \lambda_{n}}(t) \text {, for all } t \varepsilon P-\left\{t_{j_{0}}\right\}
$$

and therefore

(Note that

$$
\begin{aligned}
& \sum_{i=1}^{k} \beta_{i} \cdot \int_{P-\left\{t_{j_{0}}\right\}}\left[\left(E X_{i}\right)(t)-\left(E X_{i}\right)\left(t_{j_{0}}\right)\right] \cdot g_{t_{j_{0}}, \lambda_{n}}(t) \cdot \mu(d t) \\
& \geq \int_{P-\left\{t_{j_{0}}\right\}} g_{t_{0}, \lambda_{n}}(t) \cdot \mu(d t)>\delta \text {. }
\end{aligned}
$$

$$
\left.\left(T_{\lambda_{n}}\left(E x_{i}\right)\right)\left(t_{j_{0}}\right)=\int_{p}\left(E x_{i}\right)(t) \cdot g_{t_{j_{0}}, \lambda_{n}}(t) \cdot \mu(d t), i=1, \ldots, k .\right)
$$

However from the assumptions of the theorem, we have

$$
\lim _{n \rightarrow \infty}\left(T_{\lambda_{n}}\left(E x_{i}\right)\right)\left(t_{j_{0}}\right)=\left(E X_{i}\right)\left(t_{j_{0}}\right), \text { all } i=1, \ldots, k \text {. }
$$

Hence

$$
0=\lim _{n \rightarrow \infty}\left(\sum_{i=1}^{k} \beta_{i}\left[\left(T_{\lambda_{n}}\left(E X_{i}\right)\right)\left(t_{j_{0}}\right)-\left(E X_{i}\right)\left(t_{j_{0}}\right)\right]\right)>\delta .
$$

Thus $\delta<0$, contradicting $\delta>0$. $\square$

To show that the assumptions of Theorem 1 are not empty and they are powerful, we present

EXAMPLE 2. (i) Consider the probability space $\left([-a, a], B, \frac{\lambda}{2}-\right)$, where $a>0$, $B$ the Borel $\sigma$-algebra on $[-a, a], \lambda$ the Lebesgue measure on $[-a, a]$. Since $\frac{\lambda}{2 a}([-a, a])=1, \frac{\lambda}{2 a}$ is a probability measure on $[-a, a]$. Let also $P=\{ \pm 1, \pm 2$, $\ldots, \pm T\}$ be a finite set of integers. That is here $\omega \varepsilon \underline{0}=[-a, a]$ and $t \varepsilon P$. 
Consider the sequence of operators

such that

$$
\mathrm{T}_{\mathrm{n}}: \underline{\mathrm{B}}_{0}(\mathrm{P}) \rightarrow \underline{\mathrm{B}}_{0}(\mathrm{P})
$$

$$
\left(T_{n} X\right)(t, \omega)=X(t, \omega)\left(1-e^{-n|t|}\right)+X(-t, \omega) e^{-n|t|} \text {, for al1 } n \geq 1 .
$$

If $X \geq 0$ then $T_{n} X \geq 0$, that is $T_{n}$ is a positive operator, furthermore $T_{n}(1)=1$, for all $n \geq 1$. It is obvious that $T_{n}$ is linear.

Observe that

$$
\left(E\left(T_{n} X\right)\right)(t, \omega)=(E X)(t) \cdot\left(1-e^{-n|t|}\right)+(E X)(-t) \cdot e^{-n|t|}=\left(T_{n}(E X)\right)(t, \omega),
$$

i.e., $E T_{n}=T_{n} E$, that is $T_{n}$ is $E$-commutative for all $n \geq 1$. Therefore $T_{n}$ fulfills the assumptions of Theorem 1 .

From

$$
\left(E\left(T_{n} X\right)\right)(t)=(E X)(t) \cdot\left(1-e^{-n|t|}\right)+(E X)(-t) \cdot e^{-n|t|}
$$

it is clear that

$$
\lim _{n \rightarrow \infty} E\left[\left(T_{n} X\right)(t, \omega)-X(t, \omega)\right]=0,
$$

for all $t \in P$ and all $X \in B_{0}(P)$. Thus $T_{n}$ fulfills the conclusion of Theorem 1 .

(ii) Continuing in the setting of part (i): Let $x_{1}(t, \omega)=1, x_{2}(t, \omega)=2 t|\omega| / a$ and $x_{3}(t, w)=3 t^{2} \omega^{2} / a^{2}$. Then $\left(E x_{1}\right)(t)=1,\left(E x_{2}\right)(t)=t$ and $\left(E X_{3}\right)(t)=t^{2}$. It is obvious that $x_{1}, X_{2}, X_{3} \in B_{0}(P)$. We would like to find $\beta_{1}, \beta_{2}, B_{3}$ such that

$$
\sum_{i=1}^{3} \beta_{i}\left[\left(E X_{i}\right)(t)-\left(E X_{i}\right)\left(t_{j}\right)\right] \geq 1 \text {, for all } t \varepsilon p-\left\{t_{j}\right\} \text {. }
$$

For that we can pick $\beta_{1}$ an arbitrary real number, $\beta_{2}=-2 t$ and $\beta_{3}=1$. We have

$$
\beta_{1}(1-1)+\left(-2 t_{j}\right)\left(t-t_{j}\right)+\left(t^{2}-t_{j}^{2}\right)=\left(t-t_{j}\right)^{2} \geq 1,
$$

for all $t \in P-\left\{t_{j}\right\}$. Hence $x_{i}, i=1,2,3$ fulfill the sufficient condition of Theorem 1 .

Trivially $T_{n} X_{i}=X_{i}$, giving us $E_{n} X_{i}=E X_{i}$, for $i=1,3$. And

$$
\left(T_{n} x_{2}\right)(t, \omega)=x_{2}(t, \omega)\left(1-e^{-n|t|}\right)+x_{2}(-t, \omega) \cdot e^{-n|t|} \text {, }
$$

implying

$$
\left(E\left(T_{n} X_{2}\right)\right)(t)=t\left(1-2 e^{-n|t|}\right)
$$

Clearly

$$
\lim _{n \rightarrow \infty}\left(E\left(T_{n} X_{2}\right)\right)(t)=\left(E X_{2}\right)(t)
$$

We have seen how $x_{i}, i=1,2,3$ fulfill the assumptions of Theorem 1 .

\section{REFERENCES}

1. DUDLEY, R.M. Real Analysis and Probability, Wadsworth \& Brooks/Cole, Pacific Grove, California, 1989 .

2. HEWITT, E and STROMBERG, K. Real and Abstract Analysis, Springer-Verlag, New York/Berlin, 1965.

3. WEBA, M. Korovkin Systems of Stochastic Processes, Mathematische Zeitschrift, $\underline{192}$ (1986), 73-80. 


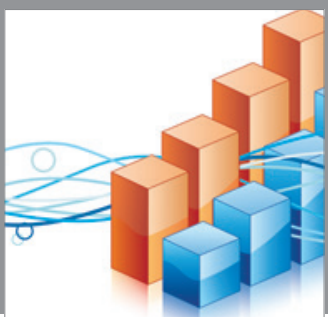

Advances in

Operations Research

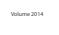

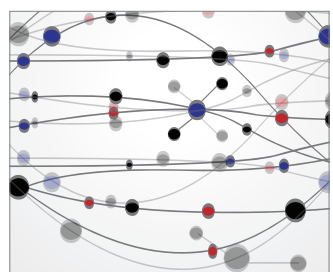

\section{The Scientific} World Journal
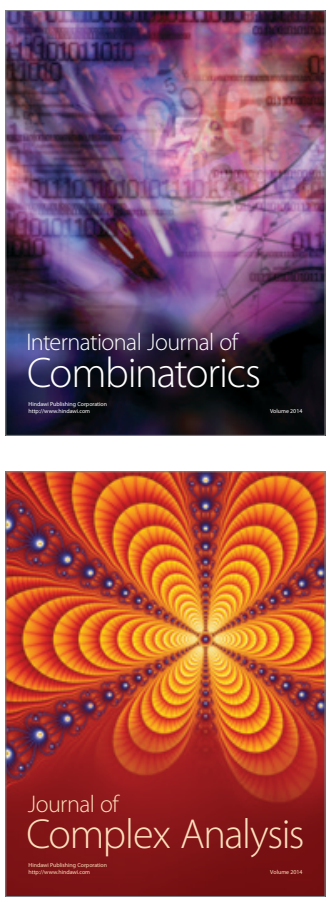

International Journal of

Mathematics and

Mathematical

Sciences
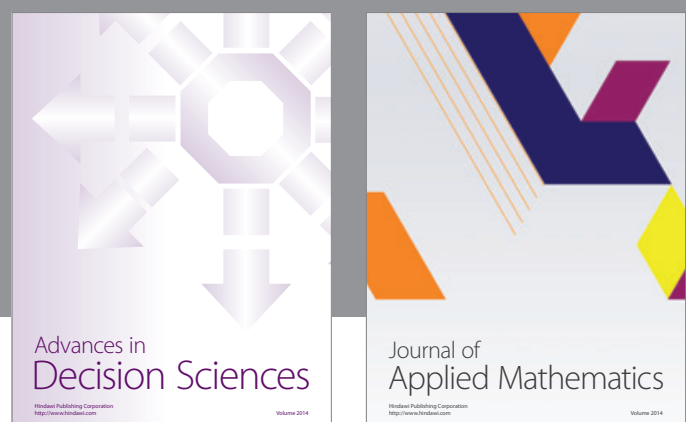

Journal of

Applied Mathematics
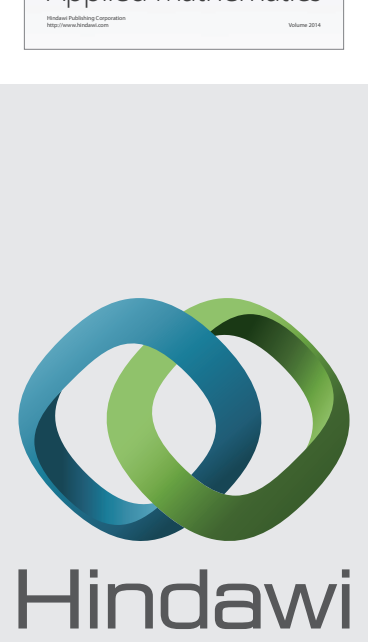

Submit your manuscripts at http://www.hindawi.com
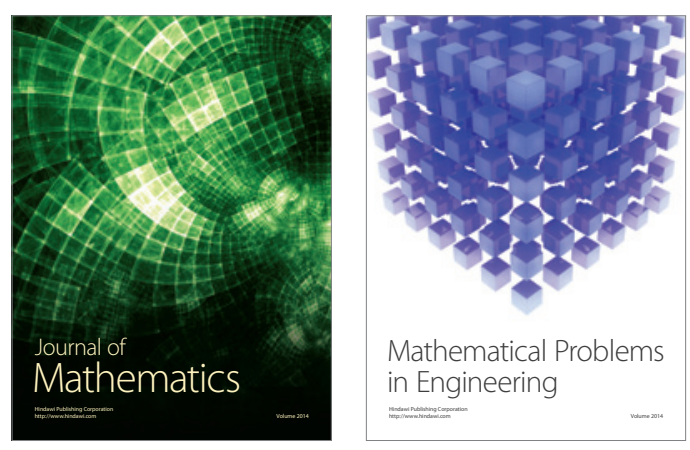

Mathematical Problems in Engineering
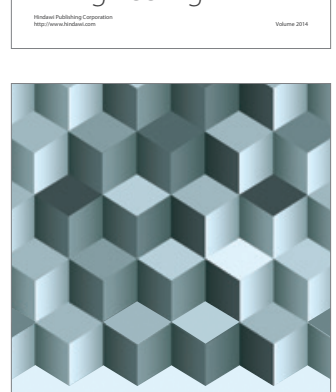

Journal of

Function Spaces
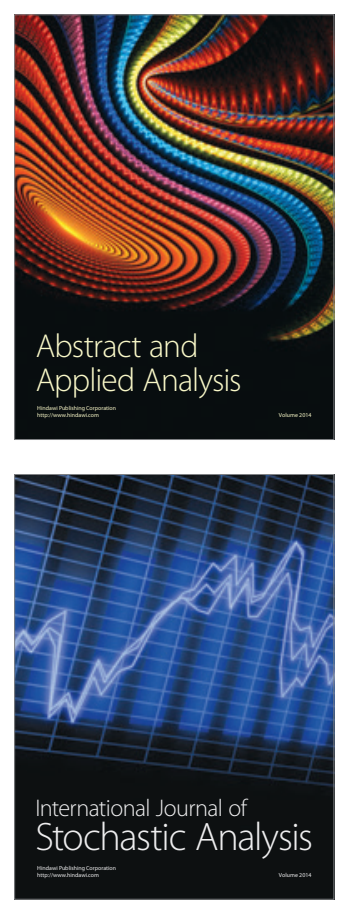

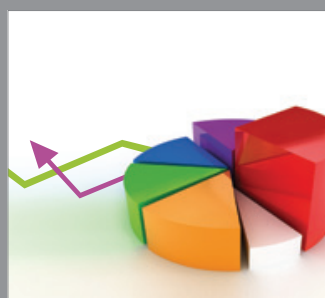

ournal of

Probability and Statistics

Promensencen
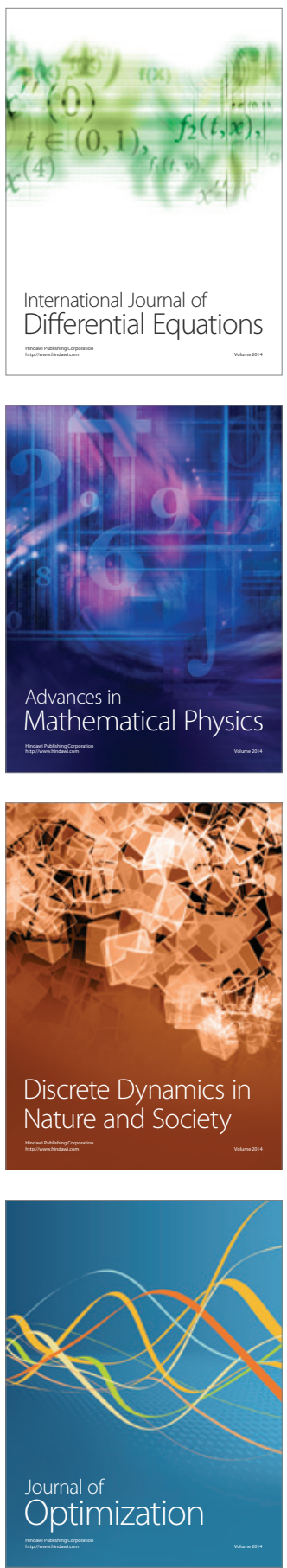